\title{
Les biotechnologies au cœur des programmes
}

\section{(2) OpenEdition}

1 Journals

Édition électronique

URL : https://journals.openedition.org/histoire-cnrs/1906

DOI : 10.4000/histoire-cnrs. 1906

ISSN : 1955-2408

Éditeur

CNRS Éditions

\section{Édition imprimée}

Date de publication : 3 juillet 2007

ISBN : 978-2-271-06558-2

ISSN : $1298-9800$

\section{Référence électronique}

"Les biotechnologies au cœur des programmes », La revue pour l'histoire du CNRS [En ligne], 17 | 2007, mis en ligne le 03 juillet 2009, consulté le 20 mai 2021. URL : http://journals.openedition.org/histoirecnrs/1906 ; DOI : https://doi.org/10.4000/histoire-cnrs.1906

Ce document a été généré automatiquement le 20 mai 2021.

Comité pour l'histoire du CNRS 


\section{Les biotechnologies au cœur des programmes}

1 Californie, 1975 : la conférence d'Asilomar rassemble des chercheurs en biologie moléculaire et en sciences de la vie qui, à partir des perspectives de développement des biotechnologies, espèrent des retombées importantes tant sur le plan industriel et économique que sociétal. Pour la première fois depuis Louis Pasteur, les biotechnologies de troisième génération font une apparition prometteuse avec une possibilité d'intervention précise et maîtrisée sur le patrimoine génétique des organismes vivants.

2 Mais entrons dans le vif du sujet : le rôle du CNRS. Était-il présent dans des initiatives telles que le programme mobilisateur «Essor des biotechnologies»? Et si oui comment? En y regardant de près, il n'y a pas eu à proprement parler de relation directe entre les initiatives du ministère et celles du CNRS. D'un côté, un programme mobilisateur «biotechnologies", de l'autre, des initiatives du CNRS dans sa politique scientifique.

3 Les actions du CNRS et du ministère n'ont pas été véritablement corrélées, exception faite, mais tardivement, des initiatives prises conjointement par le département des sciences de la vie (SDV) du CNRS, l'Inserm, l'Inra, l'Institut Pasteur, le CEA, en dehors de la direction générale du CNRS dans le cadre des groupes de recherches interorganismes (RIO) mis en place par le ministère. Pendant longtemps, le département des sciences de la vie travaillait plus avec les autres organismes qu'il ne travaillait avec les autres départements du CNRS.

4 Pour le comité de direction du CNRS, la définition des biotechnologies n'allait pas de soi. Et il n'y avait pas de véritable réflexion commune bien assumée entre les sciences de la vie, la chimie, et les sciences pour l'ingénieur (SPI). La définition est une chose, l'interprétation ou la réappropriation par le comité de direction en est une autre. La biotechnologie est une plateforme technologique qui va bien au-delà des sciences de la vie et englobe le génie des procédés, le génie chimique, les sciences de l'homme et de la société...

5 Malgré les divergences de points de vue, un certain nombre d'actions interdisciplinaires ont été lancées, programmes connotés ou ciblés biotechnologies. À 
ce titre, l'action recherche intégrée chimie/biologie a été très significative, malgré la difficulté à faire collaborer les deux départements. Mais il est vrai que les programmes interdisciplinaires se sont rarement développés dans une relation «pacifique » entre les départements impliqués. Et en particulier les départements de chimie et des sciences de la vie qui ont toujours revendiqué leur autonomie et leur personnalité propre.

Ndlr. En mars 1986, il y a eu un conflit ouvert entre le département de chimie et le département des sciences de la vie. Le contentieux provenait de la définition même du département SPI : on annonce les « sciences pour l'ingénieur » et l'on crée les " sciences physiques pour l'ingénieur ». À ce moment précis, les deux départements des sciences de la vie et des sciences chimiques n'ont pas voulu « pactiser ».

En dépit des difficultés, les programmes interdisciplinaires du CNRS ont fortement marqué la politique scientifique du CNRS dans nombre de domaines clés ou émergents. La direction générale de Pierre Papon et celle de François Kourilsky, qui leur a donné une véritable assise institutionnelle, ont été des temps forts de cette politique. Mais en ce qui concerne les biotechnologies, c'est pendant la direction générale de Serge Feneuille qui voulait avant tout renforcer les départements, qu'un des hommes forts de la direction, Jacques Demaille, directeur du département des sciences de la vie, a eu la voie libre pour mener une action qui n'était pas inter départementale. Des appels d'offre sur projets très larges et très ouverts ont été ainsi de véritables germes d'actions en biotechnologies. Sur un autre plan, l'une des responsabilités de la direction de la stratégie était l'affectation des gros budgets nécessités par les grands instruments scientifiques, en général issus de la physique mais destinés à être utilisés par un spectre beaucoup plus large de disciplines, incluant naturellement la chimie mais aussi les sciences de la vie. Au total, compte tenu de moyens « ordinaires » nettement inférieurs à ceux des laboratoires de sciences de la vie, on aurait eu tort de dire que la physique s'appropriait l'essentiel des moyens !

De la difficulté à valoriser un portefeuille de brevets

Ndlr. Qui dit " biotechnologies " dit transfert de compétences vers le milieu industriel et valorisation... Dans la course à l'excellence scientifique, le CNRS a toujours été présent. En revanche, il a fait défaut dans le transfert des compétences et dans le transfert technologique. Pierre Papon a réellement perçu l'enjeu des biotechnologies et a proposé de créer des postes d'ITA « valorisation biotechs ». La seule objection fut celle de Jean-Jacques Duby qui, d'accord sur le principe, souhaitait que la direction de la valorisation garde la décision de leur affectation. Une « défense de son territoire » et de ses "prérogatives » en quelque sorte... À ce stade de l'analyse, la question est : dans un milieu " archipellisé » comme le CNRS, quelle peut être la place d'une direction de la valorisation? Comment met-elle en valeur son potentiel brevets?

7 Ces interrogations renvoient à un problème plus large, celui du positionnement du CNRS. L'appréciation de Bernard Larrouturou en 2004 n'est pas différente de celle de François Kourilsky quinze ans auparavant. Le CNRS n'est pas une agence, c'est un organisme de recherche. Plus que distribuer les moyens, il entre dans le pilotage des laboratoires. C'est un acteur stratégique. Mais le CNRS ne balaye-t-il pas trop large ? Le CNRS ne peut pas être partout et il lui est très difficile de se positionner dans une véritable stratégie.

Ndlr. Il faut reconnaître une certaine faiblesse dans les choix stratégiques du CNRS, même si certains choix ont été plus que convaincants, comme les " actions de valorisation ", le développement de politiques scientifiques et les résultats en découlant. Dans le cadre de valorisation, c'est-à-dire de transfert de technologies, 
on ne procède peut-être pas de la même manière, et l'une des erreurs de l'« institution » CNRS, qui est d'ailleurs valable pour d'autres institutions en France, est de ne pas s'être dotée des outils nécessaires.

8 Il règne toujours cette confusion entre la preuve d'ouverture que le CNRS devrait montrer à l'égard de certaines initiatives de valorisation et l'idée qu'il puisse tout faire. Une chose est de former les chercheurs, une autre est de les aider à déposer des brevets et à valoriser leur travail. Un chercheur ne peut décemment pas tout faire, son travail de recherche et la création d'une entreprise. Une aide lui serait nécessaire dans les laboratoires pour passer du stade de la publication au stade du brevet. Il est faux de dire " à chacun son métier ", il faut trouver le moyen d'être à la fois dans la production scientifique et dans les relations avec le monde alentour. Pour un transfert de technologie efficace...

La France toujours en retard?

9 Tout ce qui précède peut expliquer qu'au cours des dix ou quinze dernières années, le CNRS n'ait pas pu jouer le rôle qu'il aurait pu jouer dans le développement des biotechs en France. On ne voit pas le CNRS dans le programme mobilisateur en 1980, on ne le voit pas plus dans le club CRIN qu'il a créé, on ne le voit pas dans des institutions comme France-Biotech ${ }^{1}$, Adebio, Généthon, l'AFM... Est-ce à dire que l'institution n'a pas les instruments pour? C'est peut-être également un problème de cloisonnement au CNRS.

10 D'après des propos recueillis le $\mathbf{1}^{\mathrm{er}}$ juin 2005 par Farid Ameur, Denis Guthleben, André Kaspi, Daniel Pardo et Girolamo Ramunni.

\section{NOTES}

1. Notons toutefois que le CNRS fait partie du comité de pilotage qui élabore le panorama des biotechnologies en France. Voir le site : http://www.france-biotech.org

\section{RÉSUMÉS}

L'histoire des biotechnologies en France est indissociable de la stratégie opérationnelle du CNRS qui vise à bâtir un socle de compétences interdisciplinaires. Jacques Sevin a longtemps été au cœur du processus d'intégration des disciplines. Il analyse ici la place et le rôle de l'établissement dans la production des connaissances et des savoir-faire issus de la révolution de la "génétique moléculaire » amorcée dans les années 1970.

The history of biotechnologies in France is connected to the operational strategy of the CNRS to build multidisciplinary skills. Jacques Sevin had been involved in the interdisciplinary 
integration process. He analyses the place and the role of the CNRS in the production of knowledge and know-how coming from the molecular genetics revolution of the 1970's. 